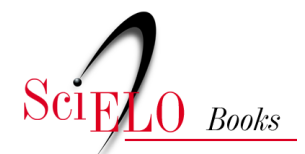

\title{
Desplazamiento lingüístico y revitalización de repertorios plurilingües en contextos de ciudad
}

\author{
Santiago Sánchez Moreano
}

\section{SciELO Books / SciELO Livros / SciELO Libros}

SÁNCHEZ MOREANO, S. Desplazamiento lingüístico y revitalización de repertorios plurilingües en contextos de ciudad. In: HABOUD BUMACHAR, M., SÁNCHEZ AVENDAÑO, C., and GARCÉS VELÁSQUEZ, F., eds. Desplazamiento lingüístico y revitalización: reflexiones y metodologías emergentes [online]. Quito: Editorial AbyaYala, 2020, pp. 183-214. Desafíos en la Diversidad collection, n. 2. ISBN: 978-9978-10541-2. http://doi.org/10.7476/9789978105726.0009.

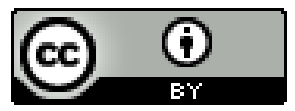

All the contents of this work, except where otherwise noted, is licensed under a Creative Commons Attribution 4.0 International license.

Todo o conteúdo deste trabalho, exceto quando houver ressalva, é publicado sob a licença Creative Commons Atribição 4.0.

Todo el contenido de esta obra, excepto donde se indique lo contrario, está bajo licencia de la licencia Creative Commons Reconocimento 4.0. 


\title{
Desplazamiento lingüístico y revitalización de repertorios plurilingües en contextos de ciudad
}

Santiago Sánchez Moreano ${ }^{1}$

\begin{abstract}
Resumen
La movilidad de grupos indígenas hacia los centros urbanos es un fenómeno en aceleración, determinado por el carácter cambiante de un mundo cada vez más globalizado. Las personas de diferentes orígenes que viven en contextos de ciudad mantienen a menudo relaciones transterritoriales y transnacionales cada vez más complejas con sus territorios de origen. Asimismo, las consecuencias de la movilidad, las cuales se desligan de la interacción relativa de múltiples factores sociales, culturales y económicos, son cada vez más visibles. Por su parte, el desplazamiento lingüístico, una de las causas de esta movilidad, ha sido ampliamente problematizado en las ciencias del lenguaje. Sin embargo, muchas veces observamos en contextos de ciudad una complejización y diversificación de las prácticas comunicativas de los hablantes. Estudiar esto supone desafíos importantes para los sociolingüistas contemporáneos interesados en comprender, desde una perspectiva posestructuralista, las prácticas de lenguaje en relación con las condiciones socio-históricas, políticas y económicas que las producen (García, Flores, \& Spotti, 2016). El objetivo de este capítulo es mostrar, a partir del caso de la ciudad de Cali (Colombia), cómo el desplazamiento y la revitalización de lenguas pueden ser reconsideradas a la luz de los conceptos de superdiversidad (Vertovec, 2007, Blommaert \& Rampton, 2011) y de des-re-territorialización (García, Flores \& Spotti, 2016), de repertorios lingüísticos socio-biográficamente constituidos (Busch, 2012, 2017) y de prácticas de lenguaje heterogéneas (Léglise, 2017).
\end{abstract}

\section{Introducción}

La movilidad de grupos indígenas hacia los centros urbanos es un fenómeno en aceleración, determinado por el carácter cambiante de un mundo globalizado que se modeliza, (re-) configura, (des-) estabiliza permanentemente. En los años 2000, el 40\% de los 30 millones de indígenas que había en Latinoamérica, es decir, 12 millo-

1 LABEX Empirical Foundations of Language / Structure et Dynamique des Langues (SeDyL). 
184

nes, habitaban en zonas urbanas (United Nations Housing Rights Programme, 2010, p. 11). Para el 2015, las estimaciones son del 49\% (Banco Mundial, 2015, p. 31). En Colombia, los datos de los censos de 1993 y de $2005^{2}$ muestran también el desplazamiento progresivo de poblaciones indígenas hacia las principales ciudades y sus periferias (Rincón Fagua \& Torres Padila, 2019, p. 68) ${ }^{3}$. Se trata de un fenómeno en aumento que representa un giro mayor en los patrones de asentamiento indígena en Colombia (Rey, 2007, p. 160).

Sus causas y consecuencias se derivan de la interacción relativa de múltiples factores sociales, culturales y económicos, propios del mundo globalizado, pero también, propios del contexto colombiano como la violencia engendrada por el conflicto interno. Sin embargo, por lo general, una de las consecuencias que más sobresale es el desplazamiento lingüístico intergeneracional en el sentido de Thomason y Kaufman (1988) y Winford (2003). No obstante, las dinámicas de la movilidad son complejas, y aunque, a primera vista causen la pérdida de una lengua, en realidad lo que generan es una diversificación de las prácticas comunicativas. Esto supone desafíos importantes para los sociolingüistas contemporáneos interesados en comprender, desde una perspectiva posestructuralista, las prácticas de lenguaje en relación con las condiciones socio-históricas, políticas y económicas que las producen (García, Flores, \& Spotti, 2016, p. 5).

La sociolingüística crítica de corte posestructuralista ofrece nuevos conceptos y herramientas metodológicas para el estudio de las prácticas comunicativas asociadas a la des-territorialización de las sociedades contemporáneas (García, Flores \& Spotti, 2016, p. 9).

2 Realizados por el Departamento Administrativo Nacional de Estadísticas de Colombia (DANE).

3 Los resultados parciales del censo de 2018 (DANE) sugieren que el 77,1\% del total de la población colombiana (48 258 494) viven en cabeceras municipales. 
Uno de estos es el de superdiversidad (Vertovec, 2007), término inicialmente utilizado para tratar de comprender el carácter disperso del transnacionalismo en los estudios migratorios en Europa, pero que ha sido utilizado para describir la "diversificación de la diversidad" en otros contextos urbanos en el mundo (Meissner \& Vertovec, 2015). La superdiversidad describe situaciones en las que el flujo de personas de diferentes orígenes, que mantienen conexiones transnacionales y transterritoriales, es cada vez más complejo y disperso (Spotti \& Blommaert, 2017, p. 169).

En contextos de superdiversidad, los repertorios lingüísticos socio-biográficamente constituidos (Busch, 2012, 2017) y las prácticas de lenguaje heterogéneas (Léglise, 2017) de los hablantes están determinados por el contacto étnico y racial, por las relaciones de poder, las ideologías lingüísticas, los aspectos socioeconómicos de las sociedades, etc.

Tanto el concepto de superdiversidad como los de repertorio lingüístico y prácticas de lenguaje heterogéneas permiten reevaluar algunas de las consecuencias de la movilidad de poblaciones, en particular, el desplazamiento lingüístico y la revitalización de lenguas. Así, el objetivo de este artículo es mostrar cómo estos dos aspectos pueden ser reevaluados a la luz de estos conceptos sociolingüísticos posestructuralistas con miras a tener una mejor comprensión de las dinámicas conurbanas asociadas al contacto lingüístico y sociocultural tomando como ejemplo la situación de diversidad y contacto de poblaciones en la ciudad de Cali.

Después de la introducción, la segunda sección describe la situación de movilidad de poblaciones indígenas hacia esta ciudad en términos de diversificación de la diversidad (Vertovec, 2007, 2009). La tercera sección describe la situación de desplazamiento tradicio- 
nalmente observada en situaciones de movilidad y lo difícil que resulta la revitalización de lenguas indígenas en estos contextos. La cuarta sección discute el giro, ya descrito en la sociolingüística posestructuralista (Léglise, 2017; Nussbaum, 2012, Spotti \& Blommaert, 2017, entre muchos otros), que conduce del análisis de "lenguas" como entidades discretas a la comprensión de los procesos biográficos y socioculturales que conllevan a la diversificación de repertorios lingüísticos y a su actualización, como prácticas de lenguaje heterogéneas, en la interacción social. La quinta sección muestra algunos ejemplos de la construcción socio-biográfica de los repertorios lingüísticos en una escuela indígena de la ciudad de Cali. La última sección intenta discutir lo que sería revitalizar, no lenguas como entidades discretas, sino repertorios plurilingües en toda su diversidad. $\mathrm{El}$ artículo termina con algunas conclusiones y perspectivas.

\section{Movilidad de grupos indígenas a Cali y diversificación de la diversidad}

En Cali, la tercera ciudad más poblada de Colombia (aprox. 1800000 habitantes, según el censo de 20184) y principal polo económico del suroccidente del país, conoce fenómenos de movilidad interna marcados por la presencia de poblaciones indígenas, afrocolombianas y rurales. Según el DANE, en el 2005, el 0,5\% de la población de Cali se reconoce como indígena. Concretamente, en la ciudad hay seis grupos indígenas sociopolíticamente organizados: los nasa, los quichua, los inga, los yanacona, los kofán y los misak

4 Por el momento, no se conocen los datos del censo para las poblaciones indígenas de Cali, por lo tanto, nos ceñiremos al censo de 2005. https://sitios.dane. gov.co/cnpv/\#!/

5 En Cali se encuentran también varios grupos indígenas que no están organizados en cabildos urbanos como los wuanan, los emberas y los pastos. Al no estar organizados, no disponen de plan de vida, por lo tanto, no son reconocidos por las 
La mayor parte viene de departamentos vecinos como Chocó, Nariño, Cauca, Putumayo y Huila, pero también de las provincias de Imbabura y Chimborazo en Ecuador (Mapa 1), por lo que la movilidad a Cali también es transnacional.

\section{Mapa 1}

Lugares de origen de los grupos indígenas de la ciudad de Cali

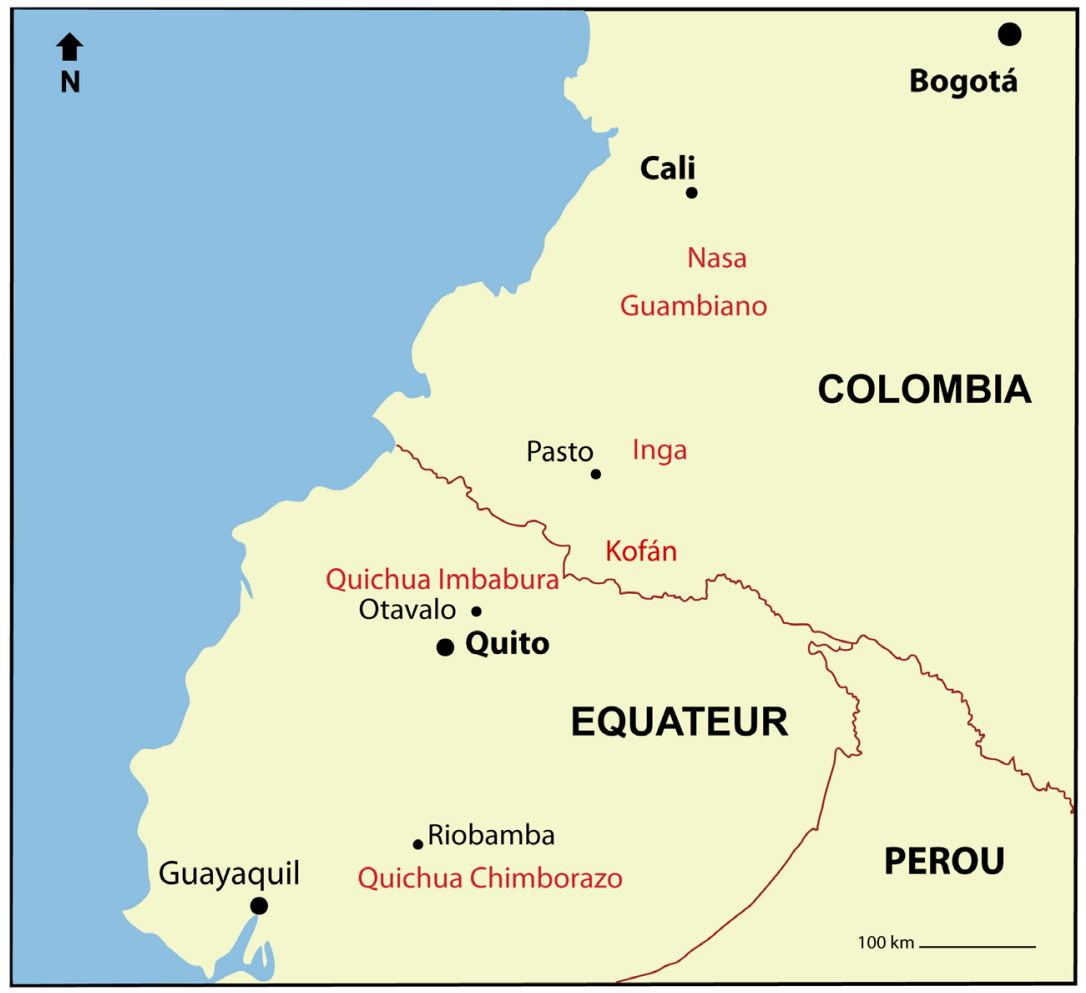

Ver Sánchez Moreano (2018, p. 36)

autoridades locales. Esto les asegura una invisibilidad absoluta en el contexto de ciudad. Sin embargo, algunos trabajos en curso dan cuenta de la presencia de pastos y embera en la Universidad del Valle (Álvarez Valencia \& Norbella Nives, 2019). 
Estos grupos están organizados en cabildos y han sido reconocidos por la Alcaldía y por el Ministerio del Interior (Motta González \& Posso, 2007). Están relativamente dispersos en diferentes sectores de la ciudad ${ }^{6}$. A pesar de los trabajos realizados y de los datos censales disponibles, sigue siendo difícil estimar el número de personas de origen indígena que llegan a las ciudades. También, es difícil estimar el número de indígenas que atraviesan las fronteras entre países (Torres, 2010, p. 10). Aun así, el censo de 2005 estima que 9466 indígenas estarían en Cali (DANE, 2005). Sin embargo, diferentes fuentes muestran una mayor variabilidad: 3635 (Motta González \& Posso, 2007), 5759 (Anacona et al., 2010), 5595 (Lara, 2013), 8634 (Planes de vida, 2014).

Esta variabilidad puede estar determinada por la dificultad técnica propia del trabajo censal, pero también por las dinámicas de las movilidades mismas. Sin embargo, gracias a los trabajos sobre los regímenes de la cuantificación sabemos que el mundo puede ser comprendido de manera objetiva a partir de cifras e indicadores. En este sentido, la cuantificación produce regímenes de conocimiento y efectos de gobernabilidad (Davis, Kingsbury, \& Engle Merry, 2012). Y en muchos casos, la ideología de la cuantificación contribuye a justificar la situación de peligro y vulnerabilidad en que se encuentran estas poblaciones, sobre todo en contextos de ciudad.

Pero más allá de la cuantificación, la presencia de poblaciones indígenas en contextos de ciudad trae consigo transformaciones socioculturales profundas que no solo afectan a las poblaciones mismas sino también a las sociedades que las reciben (Del Popolo, Oyarce,

6 Para una georreferenciación de la ubicación de los pueblos indígenas en la ciudad de Cali, referirse al trabajo etnológico de Motta González y Posso (2007) o al informe sobre bienestar de poblaciones indígenas de la alcaldía de Cali: https:// www.cali.gov.co 
\& Ribotta, 2009, p. 104). Sin embargo, estos efectos son más evidentes en las poblaciones indígenas, puesto que, por lo general, en la ciudad, son minoritarias y están minorizadas. Además de ser excluidas, sufren del peso del contacto asimétrico, el cual, está alimentado por factores ideológicos, políticos y económicos.

En los últimos veinte años, muchos trabajos han puesto en evidencia estas transformaciones socioculturales sobre todo en los grupos étnicos e indígenas minoritarios en ciudades como Bogotá (Caicedo, 2010; Martínez Bocanegra, 2008; Molina Echeverri, 2007; Ordoñez, Colmenares, Bernal \& Gincel, 2014), Cali (Barbary \& Urrea Giraldo, 2004; Motta González, 2004; Motta González \& Posso, 2007) y Medellín (Sinigüi Ramírez, 2007; Valencia Galvis, 2012, Usma Wilches, Ortiz Medina \& Gutiérrez Avendaño 2018). Muchos de estos trabajos han contribuido a la compresión de las problemáticas sociales en las ciudades (desigualdades, movilidad social y espacial, migraciones, desplazamiento forzado, violencia, etc.) desde una perspectiva histórica y contemporánea. Por lo general, las problemáticas lingüísticas y socioculturales, en esos trabajos, se postulan como estáticas, lineales y unidireccionales.

Así, se habla a menudo de fenómenos de asimilación, aculturación, desplazamiento lingüístico, integración lingüística, adaptación, etc. Sin embargo, desde la sociología, la antropología, la historia, la economía, las ciencias políticas, y más recientemente, la sociolingüística crítica, el estudio sobre las movilidades transnacionales (Vertovec, 2009) nos ha permitido pasar de tener una visión lineal y estática de los flujos migratorios a una visión más dinámica marcada por diferentes modos de movilidad, trayectorias múltiples y diferenciadas, relaciones transnacionales y transterritoriales.

Mientras que los estudios sobre migración se limitan a la descripción y análisis de la integración, la asimilación, el aprendizaje o 
190

desplazamiento de las lenguas nativas, a menudo a partir de cifras que no tienen en cuenta las dinámicas de las movilidades transterritoriales, la perspectiva transnacional y transterritorial requiere tener en cuenta la agentividad de los actores sociales en la construcción de sus propias trayectorias socio-biográficas y en su participación en diferentes comunidades de práctica (Busch, 2012).

De hecho, en sociedades superdiversas (Blommaert \& Rampton, 2011; Vertovec, 2007), las comunidades de habla, pensadas antes como entidades estables y bien delimitadas, están sujetas a cambios rápidos puesto que los hablantes participan en diferentes y variadas comunidades de práctica, muchas veces de-territorializadas (Busch, 2012, p. 505). Además, muchos de estos grupos mantienen relaciones transterritoriales de manera permanente con sus lugares y comunidades de origen a través de incesantes vaivenes, del intercambio de flujos importantes de información (incluso por internet), de intercambios económicos, materiales, inmateriales, etc. que influyen en sus prácticas de lenguaje. Todos estos aspectos no se consideran en los datos censales y en muchos de los estudios sobre migraciones, pero ponen de relieve lo que Vertovec llama la diversificación de la diversidad.

\section{Desplazamiento lingüístico y problemas de revitalización en contextos de ciudad}

Muchos de los trabajos mencionados en el apartado anterior sobre la movilidad de grupos indígenas hacia contextos de ciudad han destacado el desplazamiento lingüístico como una de las principales consecuencias de esta movilidad. Sin embargo, no existen trabajos que describan sistemáticamente y en profundidad los procesos ni las causas de este fenómeno. El desplazamiento lingüístico, es decir, la interrupción de la transmisión intergeneracional de las lenguas de primera socialización, es un fenómeno generalizado que 
191

ya ha sido revelado por los estudios realizados sobre la situación de las lenguas nativas en Colombia (Ospina Bozzi, 2015, p. 23). Este fenómeno se ve amplificado y acelerado por la urbanización de las poblaciones indígenas (Hornberger \& Coronel-Molina, 2004), por la dislocación física y social (Fishman, 1991), por factores como el peso hegemónico de las variedades mayoritarias con las que las lenguas indígenas están en contacto.

En Cali, por ejemplo, el peso hegemónico del español ha hecho que los grupos indígenas deban adaptar sus prácticas lingüísticas para expresar posturas sociales de afinidad hacia la sociedad mayoritaria utilizando formas lingüísticas características del español local (Sánchez Moreano, 2018). Esto conlleva poco a poco a la adopción de la variedad de español dominante y a la inutilidad de hablar la lengua indígena en la ciudad. Asimismo, los grupos indígenas de Cali han debido establecer una serie de estrategias de adaptación como la apropiación del espacio a través del trabajo y la reinvención de sus prácticas culturales (Motta González, 2004), no obstante, estas prácticas socioculturales no siempre están acompañadas por la práctica de sus lenguas ancestrales. Además, las nuevas generaciones de indígenas nacidos en las ciudades no necesariamente hablan las lenguas de sus padres, como lo ilustra la siguiente conversación con un indígena quichua en la ciudad de Cali: “(...) la mayoría padre como nosots aquí también hablamos solo en español entonces chiquitico nacin solo hablando español, entonce el quichua si pierde" (Cali, 2011, trabajo de campo).

Además de esto, algunos autores afirman que la educación también juega un papel importante en el desplazamiento lingüístico puesto que esta contribuye a la "castellanización" de las poblaciones indígenas y a la transmisión de los valores de la cultura dominante (Ospina Bozzi, 2015, pp. 23-24). En las ciudades, esta "castellani- 
zación” se vería aún más reforzada puesto que las poblaciones indígenas, por tener un acceso más fácil a la educación (Del Popolo et al., 2009), siguen una escolaridad que no está adaptada ni a las expectativas ni a los proyectos de vida de los pueblos indígenas en sus territorios de origen (Castellanos \& Caviedes, 2007, p. 266).

En las ciudades, las minorías indígenas escolarizadas asisten a establecimientos oficiales en los que las condiciones de implementación de proyectos etnoeducativos, que incluyen la revitalización de las lenguas, son mucho más difíciles (Rojas \& Castillo, 2005, p. 98) por haber sido concebidos para los territorios de origen ${ }^{7}$. Así, los intentos de aplicación de programas de este tipo en contextos de ciudad con poblaciones indígenas desplazadas se efectúan a través de escuelas oficiales y bajo las directrices y políticas educativas nacionales (Rojas \& Castillo, 2005). Esto hace que las acciones institucionales de la etnoeducación por lo general se inserten en las lógicas del sistema de educación nacional (Castellanos \& Caviedes, 2007, p. 269). Por lo tanto, la práctica y el aprendizaje de las lenguas indígenas en la escuela no son promovidos ni generalizados.

A pesar de esto, en algunas instituciones educativas, en contextos de ciudad, se han experimentado iniciativas de educación,

7 En los años 70 y 80, surgen programas de etnoeducación y de educación intercultural bilingüe (Calvo Población \& García Bravo, 2013; López \& Wolfgang, 1999) que tenían como objetivo crear un nuevo modelo de educación bilingüe para una educación de mayor calidad y equidad con la participación de los miembros de las comunidades (López \& Wolfgang, 1999, p. 47). Sin embargo, estos programas surgieron y fueron pensados para el medio rural (López \& Wolfgang, 1999, p. 33). La etnoeducación, como programa gubernamental, ha sido ampliamente cuestionada (Cardona \& Echeverri Restrepo, 1997; Castellanos \& Caviedes, 2007) por el hecho de que muchas poblaciones indígenas la consideran una imposición del Estado. Como contraparte, surgieron desde las comunidades mismas, numerosas iniciativas de educación propia, educación indígena y educación intercultural bilingüe (Castellanos \& Caviedes, 2007). 
venidas desde las comunidades y con el apoyo de las secretarías de educación, que intentan responder mejor a la realidad de los pueblos indígenas. Es el caso de algunas instituciones de la localidad de Bosa, al sur de Bogotá, que atienden a indígenas muiscas (Castellanos \& Caviedes, 2007, p. 271) y de la escuela integral indígena en la ciudad de Cali, de la cual hablaremos más adelante, que recibe a niños nasa, quichua, yanaconas e ingas.

Por su parte, los programas de revitalización lingüística, en los que se apoyan parcialmente las iniciativas de educación intercultural bilingüe, son numerosos en Colombia. Desde los años setenta, muchas comunidades, actores sociales e instituciones, han trabajado por el mantenimiento y la revitalización de sus lenguas a través de distintas acciones socioculturales, pedagógicas y hasta políticas (Ospina Bozzi, 2015, p. 16). Sin embargo, en su mayoría, los procesos de revitalización, al igual que los procesos de educación étnica y diferenciada, tampoco han sido pensados para los contextos de ciudad. De hecho, las iniciativas de revitalización de las lenguas de los grupos indígenas presentes en Cali han tenido lugar mayoritariamente en sus territorios de origen ${ }^{8}$.

En Cali, a pesar de que no existen programas de revitalización propiamente dichos, se encuentran iniciativas interesantes que intentan contribuir a una educación integral para los niños y niñas indígenas ${ }^{9}$. Una de estas iniciativas es la de la escuela primaria in-

8 Ver por ejemplo las iniciativas de revitalización del Consejo Regional Indígena del Cauca - CRIC (Pancho \& Navas, 2007), la creación de una universidad indígena en el departamento del Cauca (https://uaiinpebi-cric.edu.co/), la creación de materiales y el desarrollo de programas de etnoeducación, también en este departamento, con lenguas como el nasa yuwe (Sierra Martínez et al., 2015) y el nam trik (Díaz Montenegro, Rojas Curieux, \& Gonzáles Castaño, 2011), entre otros.

9 Según datos de la Secretaría de Educación de Cali, en la ciudad habría 26 instituciones educativas que aplican programas de etnoeducación. También, hay 
194

tegral indígena que recibe a estudiantes, mayoritariamente de los pueblos nasa, yanacona y quichua que viven en el centro de la ciudad. Esta escuela, adscrita al Colegio Santa Librada de Cali, integra en su currículo algunos contenidos socioculturales y de lenguas indígenas. Los niños reciben una educación clásica basada en los programas oficiales, pero con un énfasis en la interculturalidad, en los que se incluyen temas como la danza, la música, la relación con la tierra y la enseñanza de lenguas, en particular, el quichua y el nasa yuwe. Desafortunadamente, una vez terminada la educación primaria e iniciada la educación secundaria, el programa no tiene continuidad y los estudiantes retoman una educación clásica.

Lejos de estar centrado en la revitalización de lenguas, este programa se inscribe en la línea de la etnoeducación y está focalizado en la inclusión de contenidos socioculturales dirigidos a varios grupos étnicos (los quichuas, ingas, nasa, yanaconas, etc.) que comparten un mismo espacio sociocultural: la escuela. Allí, al igual que en la ciudad donde los grupos interactúan entorno a actividades económicas o a la organización de eventos culturales, los niños y niñas de los grupos indígenas interactúan entre sí en torno a actividades escolares, extracurriculares y socioculturales.

Los grupos indígenas de Cali no pueden entenderse como entidades autónomas y separables, comunidades aisladas o comunidades de habla, sino como grupos heterogéneos que interactúan entre sí permanentemente. Esto necesariamente tiene incidencias en la forma como se constituyen sus repertorios lingüísticos en la complejidad del espacio-tiempo (Blommaert, 2015) que constituye la escuela, a partir

programas de investigación universitarios que buscan establecer lineamientos y llevar a cabo acciones para promover una educación multicultural en la ciudad de Cali (https://www.javerianacali.edu.co/intercultural/investigacion-aplicada/ educacion-intercultural). 
de diferentes materiales lingüísticos (lenguas, registros, dialectos, formas de hablar, etc.) a los que están expuestos. Según Rampton (2006, citado por Spotti \& Blommaert, 2017, pp. 164-165), estos materiales tienen sus orígenes, no en procesos extensos de aprendizaje y socialización de lenguas, sino en contactos, superficiales y esporádicos, a veces más intensos, que tienen lugar en ambientes de aprendizaje informales, en grupos inter-generacionales o en grupos que comparten los mismos intereses socioculturales, dentro y fuera de la escuela.

Esta dinámica de la interacción social que podemos ver en la escuela, aunque no exclusivamente, nos lleva a cuestionar la revitalización de lenguas en este contexto y a considerar más bien la promoción de ambientes de interacción multilingües y multiculturales. Sin embargo, esto significa hacer el ejercicio de des-construir lo que consideramos que es una "lengua".

\section{De las "lenguas" a los "repertorios plurilingües"}

En los últimos años, numerosos trabajos en antropología lingüística, en sociolingüística crítica y en lingüística aplicada han venido des-construyendo, de manera crítica y desde una perspectiva posestructuralista, el concepto de "lengua". Una lengua, argumentan, es una construcción o invención ideológica, normativa y sociohistórica (Léglise, 2018; Makoni \& Pennycook, 2005; Otheguy, García \& Reid, 2015) que ha contribuido a la instauración de fronteras lingüísticas. En este sentido, las lenguas, variedades de lengua, dialectos, registros o estilos y otras formas de hablar pueden ser definidas por criterios como la afiliación social, política o étnica de sus hablantes, por políticas lingüísticas nacionales o regionales impuestas en un determinado territorio, por razones sociohistóricas e ideológicas, pero no por cuestiones netamente lingüísticas (Otheguy et al., 2015; Romaine, 2000). 
Los efectos de estos criterios ideológicos, políticos, normativos y sociohistóricos se han materializado en la forma cómo hemos entendido las lenguas, en la manera cómo se han construido las políticas lingüísticas y educativas, y, sobre todo, en cómo la gente ha llegado a identificarse socioculturalmente con las "lenguas" (Makoni \& Pennycook, 2005, p. 138). Nosotros mismos, como lingüistas, hemos contribuido a la creación de fronteras lingüísticas al crear y delimitar nuestros corpus, incluso plurilingües, cuando en la realidad de la interacción los hablantes suelen transgredir dichas fronteras (Léglise, 2018).

A pesar de que sigue siendo una tarea compleja hablar de lo heterogéneo de las prácticas plurilingües de los hablantes sin recurrir artificialmente a la delimitación de fronteras (Léglise, 2018, p. 2), la des-construcción de "lenguas" nos ha llevado a idearnos múltiples maneras de explicar los fenómenos lingüísticos y sus consecuencias sociales alejándonos de la idea tradicional de lengua.

Jørgensen et al. (2011), por ejemplo, sugieren centrar el análisis, no en las lenguas, sino en los linguistic features, es decir, los "recursos lingüísticos" (en la terminología de Blommaert y Backus (2011)) que están asociados socioculturalmente a diferentes lenguas y formas de hablar que los hablantes utilizan en el transcurso de la interacción y en función de sus necesidades comunicativas y del contexto sociocultural.

Asimismo, basada en los trabajos de Boutet et al. (1976) sobre la noción de pratiques langagières, Léglise $(2013,2018)$ sigue esta misma perspectiva, pero prefiere hablar de pratiques langagières heterogènes. En esta noción, el adjetivo langagière se distingue de linguistique (pratique linguistique) en cuanto no se hace referencia a entidades discretas, es decir, a lenguas, sino más bien a la actividad social de utilizar el lenguaje. Por su lado, el adjetivo hétérogène busca abarcar 
los fenómenos de variación lingüística y de pluri-acentuación (Léglise, 2017, p. 254), es decir, la variabilidad de las formas lingüísticas y la diversidad de acentos con los que hablan las personas. En la idea de pratique o práctica se comprende la imbricación del uso del lenguaje que hacen los hablantes en el mundo social. Así, una pratique langagière es fundamentalmente una práctica social puesto que hace parte del conjunto de prácticas sociales de los individuos y, por tanto, contribuye a la construcción del mundo social, al mismo tiempo que está determinada por él (Boutet et al., 1976).

En español, podríamos hablar de "prácticas lingüísticas", incluso de "prácticas multilingües" o "translingües", pero caeríamos en el mismo error al referirnos al uso de lenguas como entidades discretas. Por eso, podríamos optar por "prácticas de lenguaje heterogéneas" para describir la diversidad de recursos lingüísticos a disposición de los hablantes y que están socioculturalmente asociados a diferentes lenguas, dialectos, acentos, variedades diatópicas, registros, estilos, géneros, etc.

Tanto la perspectiva de Jørgensen et al. como la de Léglise, entre muchas otras, son visiones en las que lo que resulta importante, no son las "lenguas", sino la práctica de los variados recursos que hacen parte los repertorios lingüísticos (Gumperz, 1964) de los hablantes. En este sentido, en la interacción social, los hablantes utilizan, sobre todo y principalmente, recursos lingüísticos, más que lenguas en el sentido de "paquetes" o sistemas coherentes (Spotti \& Blommaert, 2017, p. 168) o entidades discretas y delimitables.

Ahora, las prácticas de lenguaje heterogéneas son posibles gracias a la existencia de repertorios lingüísticos heterogéneos, es decir, repertorios plurilingües, pluridialectales, pluriestilísticos, pluriacentuales. Un repertorio lingüístico es el conjunto que incluye "todas 
198

las formas de hablar" que los hablantes conocen, saben cómo usar, y utilizan en un encuentro comunicativo o espacio sociocultural determinado, que pueden ser lingüísticos (variedades lingüísticas) y socioculturales (géneros, estilos, voces, etc.) (Spotti \& Blommaert, 2017, p. 171). Los repertorios se constituyen a partir de la experiencia vivida del lenguaje (Busch, 2017), es decir, en las trayectorias individuales y colectivas de cada una de las personas.

En este sentido, el conocimiento de una "lengua" depende básicamente de la trayectoria socio-biográfica de una persona. Esto significa que los repertorios no se desarrollan o construyen en una perspectiva lineal, sino más bien, de manera dinámica y aleatoria de acuerdo con las necesidades situacionales y comunicativas que una persona puede encontrar a lo largo de su vida (Spotti \& Blommaert, 2017, p. 171), en la interacción con los demás y a través de su participación en diversos espacios, grupos socioculturales o comunidades de práctica.

Así, los repertorios lingüísticos se forjan gracias al contacto de poblaciones de diversos orígenes étnicos, a las migraciones, la movilidad espacial y social, a las relaciones transnacionales y transterritoriales, a las diferencias culturales, al estatus socio-económico de los individuos, las relaciones de poder entre los diferentes grupos, al prestigio de las lenguas, a las representaciones sociales que los hablantes tienen de ellas, y a las ideologías y políticas lingüísticas impuestas desde arriba, pero a veces también desde abajo. Es decir, a través de todos los aspectos que caracterizan una sociedad superdiversa.

Busch afirma que en situaciones de superdiversidad los sujetos están moldeados y constituidos en su pensamiento, en su forma de hablar y de actuar, en sus sentimientos y deseos por el poder, a veces normativo y restrictivo, de categorías sociales discursivamente establecidas. Los hablantes crecen expuestos a varias lenguas, varie- 
dades de lengua o incluso a variedades estilísticas, registros, formas particulares de hablar, acentos, etc., y a las creencias, valores e ideologías lingüísticas (Kroskrity, 2006) que están ligadas a cada una de esas formas de hablar. Estas formas de hablar, recursos lingüísticos o linguistic features vienen a ser constitutivos de sus repertorios plurilingües y se caracterizan por ser híbridos, translingües, plurales e inestables (Spotti \& Blommaert, 2017, pp. 171-172).

Según Spotti y Blommaert (2017), esta perspectiva de los repertorios lingüísticos cuestiona las nociones de bilingüismo y multilingüismo, remplazando la creencia de la "full competencia" en dos o más lenguas por una visión más dinámica en la que los hablantes utilizan múltiples recursos disponibles con diferentes grados de fluidez. En el caso de Cali, veremos que estos repertorios pueden ser complejos y heterogéneos puesto que la situación de contacto de poblaciones es también compleja y responde a lo que se describe para los contextos de superdiversidad.

\section{Repertorios lingüísticos heterogéneos en Cali}

A través de una actividad de descripción de repertorios lingüísticos realizada en la escuela integral indígena de Cali, basada en los trabajos de Busch $(2012,2017,2018)$, se les pidió a los estudiantes que describieran las "lenguas" que han hecho parte de sus vidas o que han jugado un papel importante a través de colores en una silueta humana ${ }^{10}$. Las representaciones de los estudiantes muestran que sus

10 Busch utiliza una perspectiva posestructuralista, multimodal y biográfica para describir empíricamente por medio de siluetas y representaciones verbales, la experiencia vivida del lenguaje. Para el detalle de los aspectos metodológicos de este tipo de ejercicio, el lector puede referirse a Sánchez Moreano (2019), y de manera más general a los trabajos teóricos y metodológicos de Busch (2012, 2015 y 2018). 
repertorios lingüísticos no se reducen únicamente al conocimiento del español como podría indicarnos el desplazamiento lingüístico descrito por la mayoría de trabajos. Por el contrario, vemos cómo sus repertorios son heterogéneos y se han diversificado en la ciudad, reflejando la diversidad que genera el contacto de poblaciones.

Al preguntarle a Yani, del pueblo nasa, por la representación de su repertorio él responde (Ilustración 1):

Pues yo hablo nasa-yuwe porque, mejor dicho, en mi comunidad siempre me hablan como ese idioma, tonce, ese es el que yo más sé, tonce por eso yo utilicé el verde sobre la naturaleza, por eso lo pinté de verde, sobre las montañas. Nasa-yuwe es como yo sé hablar $\mathrm{y}$ pronunciar $\mathrm{y}$ un poquito escribir.

Sobre el castellano, dice: "Porque es el idioma desde que llegué a la ciudad, aprendí (...) porque sabía más el nasa-yuwe”. Y sobre las demás lenguas, afirma: "el inglés lo aprendí aquí pues un poquito porque, nos enseñaba la profesora y ahí jue aprendiendo poco a poco un poquito inglés. El quichua también como la profesora M...nos enseña, nos saluda, tonce ahí voy aprendiendo poco a poco”.

Yani declara conocer cuatro lenguas, una de primera socialización: el nasa yuwe; una de segunda socialización: el español; y dos de aprendizaje escolar: el inglés y el quichua. Esta última es también lengua de contacto, pues Yani interactúa permanentemente con niños quichuas en la escuela. El aprendizaje del inglés hace parte de los contenidos oficiales del programa de educación y el aprendizaje del quichua hace parte de los contenidos etnoeducativos que se implementan allí. El hecho de que Yani haya decidido representarlos en su silueta muestra los esfuerzos de los profesores por incluir las lenguas indígenas en la educación y por conjugar su enseñanza con los programas de educación oficiales. 


\section{Ilustración 1 \\ El retrato lingüístico de Yani}

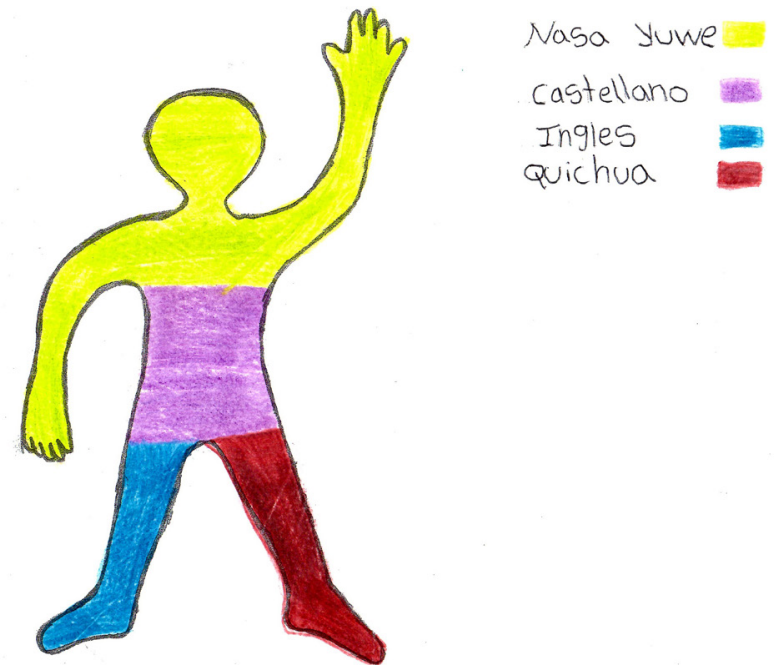

Fuente: Trabajo de campo, Cali 2016.

Un análisis más profundo de las explicaciones de Yani sobre su representación podría arrojarnos luces sobre la manera como él percibe el lenguaje en la ciudad. Por ejemplo, el paso del nasa yuwe al español que Yani ha experimentado al llegar a la ciudad puede ser interpretado como una metáfora de la movilidad desde su territorio de origen hacia la ciudad de Cali. Y el aprendizaje del inglés y del quichua como una apertura hacia otras culturas tanto mayoritarias como minoritarias. De manera interesante, estas no son las únicas "lenguas" que aparecen en los repertorios declarados por los estudiantes de esta escuela. Kate (Ilustración 2), quien manifiesta no pertenecer a una comunidad indígena, declara conocer el quichua, el nasa yuwe y el inglés, lenguas que ha experimentado de cerca en la escuela. Pero, además, manifiesta conocer algo de francés. 


\section{Ilustración 2 \\ El retrato linguiístico de Kate}
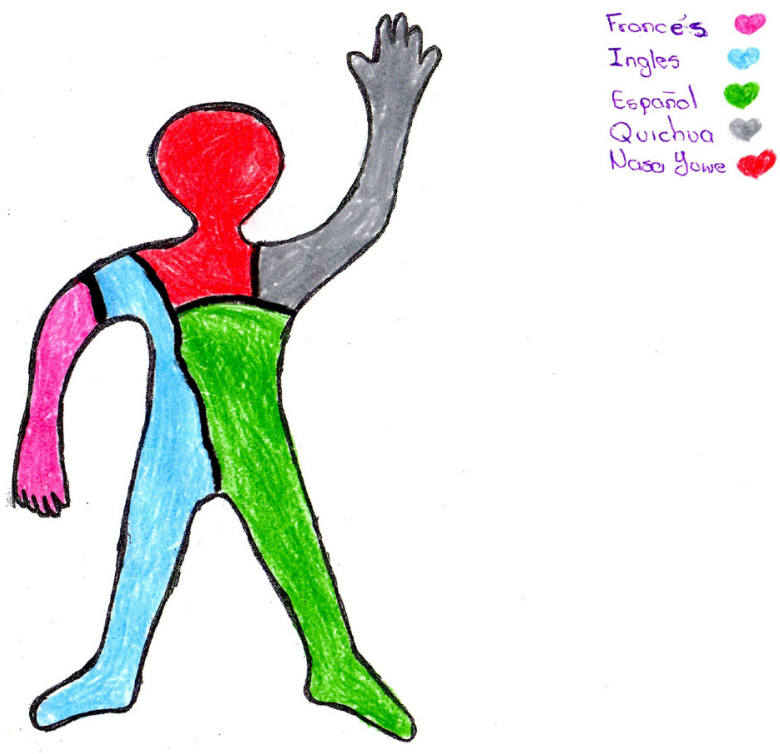

Fuente: Trabajo de campo, Cali 2016.

Al preguntarle por esto, Kate explica: “yo hablo español y cuando yo iba donde trabajaba mi mamá, la patrona de ella me enseñaba a hablar francés (...) y la profesora me enseñaba inglés, y la otra profesora me enseñaba quichua”. Estas declaraciones resultan interesantes en el sentido que, como lo afirma Busch, las prácticas de lenguaje de los hablantes, además de ser des-territorializadas, dan cuenta de los contactos que experimentan los hablantes con diferentes personas a lo largo de sus vidas. En este sentido, las lenguas internacionales pueden volverse objeto de deseo como en el caso del francés o el inglés, probablemente por la ideología asociada al éxito social que implica conocer estas lenguas. Por ejemplo, Claudia 
(Ilustración 3), una chica nasa, pintó el inglés en el lado del corazón y a este respecto, afirma: "el inglés es lo que yo quiero hablar más" a pesar de que "el inglés la profesora nos la ha enseñado un poquito".

Ilustración 3

El retrato linguíístico de Claudia

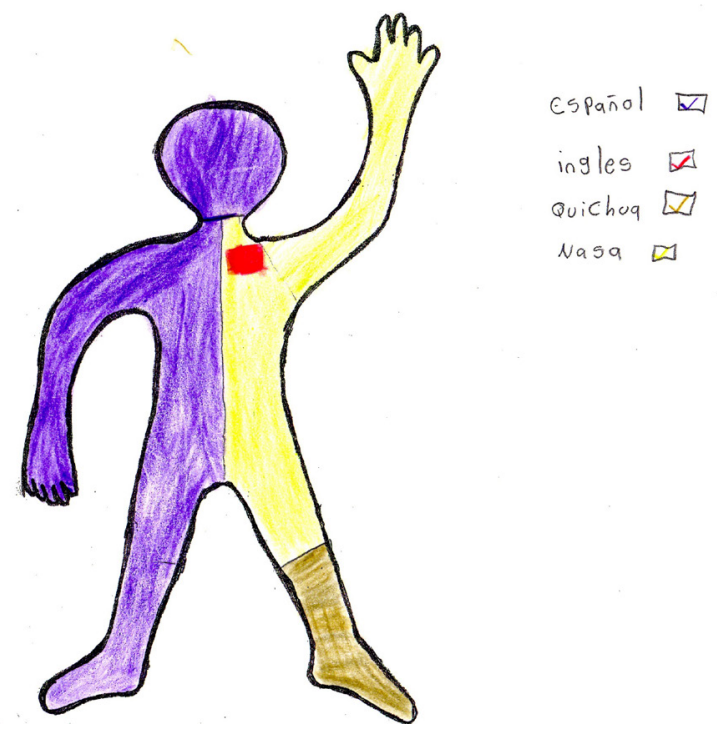

Fuente: Trabajo de campo, Cali 2016.

La presencia del francés y del inglés como lenguas internacionales muestra también la exposición a la occidentalización que experimentan los niños indígenas en la ciudad. Al conocer el prestigio de estas lenguas y las actitudes positivas que tienen generalmente los estudiantes hacia ellas, reconocemos las relaciones de poder y las ideologías lingüísticas que se reproducen en la escuela y en la ciudad. Pero también, se pone de relieve la pertinencia de incluir lenguas indígenas en los programas de educación en la escuela como forma de equilibrar esas relaciones de poder. 
Finalmente, y de manera sorprendente, aparecen en la descripción de repertorios lingüísticos, lenguas "distantes" que tradicionalmente no hacen parte del paisaje lingüístico de la ciudad de Cali. Sabri, por ejemplo, pintó en su silueta el coreano.

\section{Ilustración 4 \\ El retrato linguiístico de Sabri}

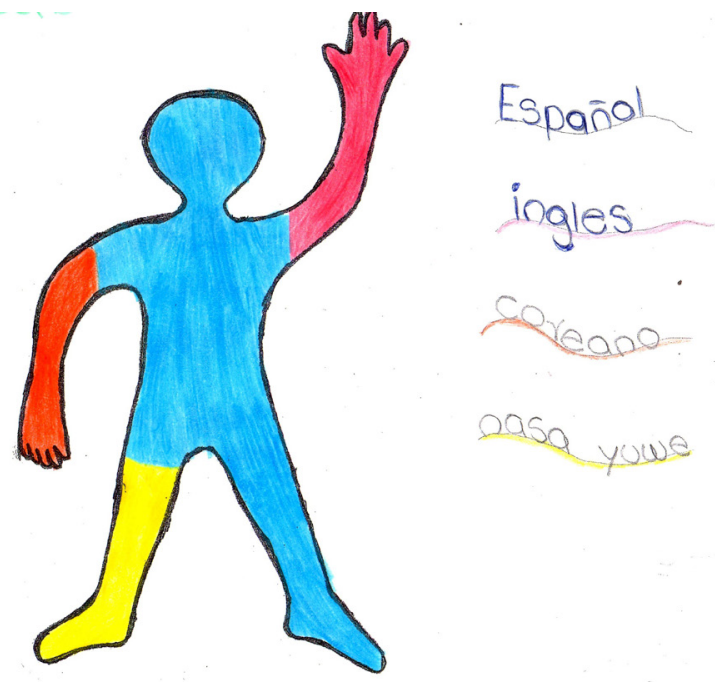

Fuente: Trabajo de campo, Cali 2016.

Al preguntársele por qué, Sabri afirma: "el coreano, porque yo miraba drama oramas (...) yo miro doramas, es como una novela, pero en coreano, a mí me parece más fácil aprender una lengua viendo en la tele". Estas teleseries coreanas para adolescentes (i.e. Doramas), no las transmiten en la televisión local, sino en Internet. Sabri nos dice que una búsqueda simple en internet da acceso a estas teleseries: “(...) eh, o sea, en el Internet uno pone drama fever y aparecen doramas". 
Este ejemplo, además de no ser aislado, ilustra el papel que puede jugar Internet y las tecnologías de la comunicación en la constitución de repertorios lingüísticos. Como lo afirman Androtsopolous y Juffermans (2014, p. 1): “comprender la relación entre el lenguaje y las trayectorias individuales en contextos superdiversos parece imposible si no se considera la comunicación digital”. Para ellos, las funciones de la conectividad digital individual son múltiples: "familias transnacionales que hablan por Skype, parejas que mantienen flujos de conversación a través de textos [iy, más recientemente Whatsapp!], migrantes indocumentados que trazan sus rutas a través de teléfonos móviles"; y, como lo ilustra el ejemplo de Sabri, adolescentes que aprenden lenguas geográficamente lejanas a través de series que encuentran en Internet. Como dicen estos autores, las implicaciones de estas trayectorias para los repertorios lingüísticos de los individuos y su diversificación son múltiples (2014, p. 1), más aún, cuando los discursos sobre la revitalización de lenguas en peligro (Duchêne \& Heller, 2008) son cada vez más frecuentes.

\section{Discusión: hacia una revitalización de repertorios plurilingües}

Las dinámicas de movilidad, el contacto de poblaciones, las relaciones de poder y el peso de las ideologías lingüísticas observables en Cali, se pueden asimilar a la "diversificación de la diversidad" que ha sido descrita para contextos superdiversos en el mundo. Busch (2012), al criticar el concepto gumperziano de "comunidades de habla", afirma que las prácticas de lenguaje son a menudo desterritorializadas y que los hablantes participan más bien en variadas y diversas comunidades de práctica. Esto nos lleva a revisar no solo los conceptos de migración, desplazamiento lingüístico y revitalización de lenguas, sino también las dicotomías urbano-rural y nacional- 
transnacional, en el sentido en que los espacios geográficos están cada vez más interconectados (B. Makoni, 2017) y que las dinámicas lingüísticas son cada vez más transterritoriales.

Esta mise en question tiene consecuencias importantes en la forma cómo comprendemos los repertorios lingüísticos de los hablantes y en la forma cómo estos se diversifican en situaciones de superdiversidad, como lo ilustran las representaciones de los niños y niñas de la escuela integral indígena de Cali descritas en la sección 5.

En este sentido, la revitalización de una o varias lenguas indígenas en esta escuela, podría ser problemática si no se tienen en cuenta los diferentes aspectos que entran en diversificación de los repertorios lingüísticos de los hablantes. En términos de políticas lingüísticas, por ejemplo, habría que preguntarse qué variedad de lengua, entre las que están presentes en la escuela, habría que revitalizar, asumiendo que exista una variedad normalizada. Decidir esto implica ya un acto de imposición y por lo tanto un acto de poder. Los quichuas ecuatorianos en Cali, por ejemplo, vienen de dos provincias diferentes del Ecuador. Hablan dos variedades de quichua mutualmente entendibles, pero con marcas de diferenciación reivindicadas por los mismos hablantes. Además, los grupos indígenas del Putumayo y Nariño que se han reagrupado en el cabildo urbano inga de Cali, también hablan otra variedad de quechua, el inga. Por su parte, los yanaconas se identifican como descendientes de los incas y de la cultura y la lengua quichua. En este sentido, sería extremadamente difícil revitalizar "el quichua" sin tener que tomar decisiones de tipo político e ideológico privilegiando una variedad por encima de las otras, y sin llevar al silencio y a la exclusión las otras variedades y a sus hablantes. Algo similar podría suceder con la población nasa de Cali puesto que sus integrantes provienen de 
diversas localidades (resguardos) ubicadas en tres departamentos del suroccidente colombiano: Cauca, Huila y Valle.

Además, en Cali, solo seis grupos indígenas han sido reconocidos por las autoridades. Este reconocimiento les ha brindado el estatus necesario para llevar a cabo acciones de desarrollo y bienestar social en favor de sus comunidades. Pero no es el caso de las muchas otras poblaciones indígenas, también presentes en la ciudad, algunas escolarizadas, pero de las cuales se sabe muy poco puesto que aún no han logrado ese reconocimiento.

En vista de eso, es aquí donde podemos pensar en revitalización, pero no de lenguas de manera aislada, sino de repertorios plurilingües en los que todas las "lenguas" (y también las diferentes formas de hablar, voces, registros, dialectos, acentos, variedades regionales, incluso idiolectos, etc. que convergen en un espacio social como la escuela) puedan tener una voz y salgan del silencio y de la invisibilidad a los que han sido reducidos (Léglise, 2017, p. 259) en contextos de ciudad. Esto implicaría, por un lado, integrar todas las lenguas y variedades minorizadas en la escuela, un reto mayor para los sistemas educativos (etnoeducación, educación intercultural bilingüe, etc.) y, en general, para la investigación en los países del Global South (Léglise, 2017, p. 258). Por otro lado, implicaría visibilizar y valorar las prácticas de lenguaje heterogéneas de los hablantes a través de diferentes actividades. Léglise (2017) nos provee varios ejemplos interesantes de iniciativas que se pueden llevar a cabo: 1) la valorización de prácticas de lenguaje heterogéneas en la escuela; 2) la elaboración de pedagogías críticas para desarrollar una conciencia meta-literatizante de modo que los estudiantes se vuelvan conscientes del lugar que ocupan en el mundo y de lo que pueden hacer en él (Alim, 2004); 3) la crítica de las prácticas hegemónicas que han contribuido a formar sus experiencias y percepciones para liberarlas de las ideologías, de 
las estructuras y de las prácticas dominantes" (Morrell \& DuncanAndrade, 2004, p. 250); 4) la visualización de diferentes formas de hablar (lenguas, dialectos, acentos, registros, etc.), habitualmente invisibles, con el objetivo de subvertir los mecanismos de naturalización, de reproducción y de legitimación de desigualdades sociales y raciales en la escuela (Léglise, 2017; Martín Rojo, 2015).

Dichas acciones contribuirían ampliamente a la revitalización de repertorios plurilingües dándoles una mayor difusión y equidad a las formas de habla minorizadas, por lo menos en la escuela. Finalmente, esto contribuiría, no solo a la promoción de un multilingüismo sostenible, sino también a una valorización de repertorios y prácticas de lenguaje heterogéneas por parte de las poblaciones mismas, un criterio importante para que las "lenguas" vivan.

\section{Conclusiones}

La movilidad de poblaciones indígenas y rurales hacia la ciudad de Cali, lejos de ser estática y lineal, es dinámica y contribuye a la diversificación de la diversidad. Los fenómenos que se desligan de esta diversificación son también dinámicos y complejos como lo muestran las representaciones de los repertorios lingüísticos de los estudiantes de la escuela integral indígena de Cali. En este contexto, el desplazamiento lingüístico y los procesos de revitalización deben ser reconsiderados a la luz de esta complejidad y superdiversidad. Para esto, la sociolingüística posestructuralista provee un marco de análisis y metodológico interesante que nos permite des-construir la idea de que las lenguas son entidades discretas. También, nos permite comprender cómo se constituyen los repertorios lingüísticos en las trayectorias socio-biográficas de los hablantes y a través del contacto con los demás, incluido los intercambios comunicativos por Internet. Sin embargo, queda mucho trabajo por hacer, como por 
ejemplo pasar de la descripción de repertorios heterogéneos a la descripción de prácticas de lenguaje heterogéneas socialmente situadas observables en el día a día. En este sentido, pensamos que el giro que representa la sociolingüística crítica posestructuralista para el estudio del lazo existente entre el lenguaje y el mundo social en contextos de superdiversidad es más que ineludible.

\section{Referencias bibliográficas}

Alim, H. S. (2004). You Know My Steez: An Ethnographic and Sociolinguistic Study of Styleshifting in A Black American Speech Community. Durham, N.C.: Duke University Press.

Álvarez Valencia, J. A. y Norbella Nieves, M. (5-9 de agosto de 2019). Diversidad, reconocimiento e inclusión: Perfil sociolingüístico y académico de los estudiantes indígenas de pregrado de la Universidad del Valle - Sede Cali. Ponencia presentada en la II Semana Cultural de las Lenguas Indígenas. Universidad del Valle, Cali.

Anacona, A., Cardona, M. I., \& Tunubala, M. (2012). Estudio de Caracterización de Pueblos Indígenas: Kofán, Misak-Guámbianos, Quichuas, Ingas, Yanaconas, Nasas habitantes de Santiago de Cali. [Informe de proyecto: "Asistencia Técnica para la Implementación de la Política Pública Indígena en Santiago de Cali"]. Cali: Alcaldía de Santiago de Cali.

Androutsopoulos, J., \& Juffermans, K. (2014). Digital language practices in superdiversity: Introduction. Discourse, Context \& Media, 4-5, 1-6. https:// doi.org/10.1016/j.dcm.2014.08.002

Banco Mundial (2015). Latinomérica Indígena en el Siglo XXI. Washington, D.C.: Banco Mundial.

Barbary, O., \& Urrea Giraldo, F. (2004). Gente negra en Colombia: Dinámicas sociopoliticas en Cali y el Pacifico. Cali, [Colombia]/Paris, [France]: Centro de Investigaciones y Documentación Socioeconómicas, Universidad del Valle, IRD, COLCIENCIAS.

Blommaert, J., \& Backus, A. (2011). Repertoires revisited: "Knowing language" in superdiversity. Working Papers in Urban Language \& Literacies, 67, 1-26.

Blommaert, J. (2015). Chronotopes, Scales, and Complexity in the Study of Language in Society. Annual Review of Anthropology, 44(1), 105-116. https://doi.org/10.1146/annurev-anthro-102214-014035 
Blommaert, J., \& Rampton, B. (2011). Language and Superdiversity. Diversities, 13(2), 1-21.

Boutet, J., Fiala, P., \& Simonin-Grumbach, J. (1976). Sociolinguistique ou sociologie du langage? Critique, 344, 68-85.

Busch, B. (2012). The Linguistic Repertoire Revisited. Applied Linguistics, 33(5), 503-523.

(2017). Expanding the Notion of the Linguistic Repertoire: On the Concept of Spracherleben. The Lived Experience of Language. Applied Linguistics, 38(3), 340-358. https://doi.org/10.1093/applin/amv030

(2018). The language portrait in multilingualism research: Theoretical and methodological considerations. Working Papers in Urban Language \& Literacies, 236, 1-13.

Caicedo, L. P. (2010). Los kichwa-otavalos en Bogotá. En A. Torres (Ed.), Niñez indígena en migración Derechos en riesgo y tramas culturales (pp- 139226). Quito: FLACSO, UNICEF, AECID.

Calvo Población, G. F., \& García Bravo, W. (2013). Revisión crítica de la etnoeducación en Colombia. Historia de la educación: Revista interuniversitaria, 32, 343-360.

Cardona, M., \& Echeverri Restrepo, J. A. (1997). Etnoeducación y cultura: Elementos para una caracterización de la educación indígena en el departamento del Amazonas. Boletín de Antropología Universidad de Antioquia, 11(28), 71-92.

Castellanos, M., \& Caviedes, M. (2007). La educación en los pueblos indígenas de Colombia. En Rey, E. (Ed.). Indígenas sin derechos. Situación de los derechos humanos de los pueblos indígenas (pp.265-282). Bogotá: Centro de Cooperación al Indígena: Organización Indígena de Antioquia.

Davis, K. E., Kingsbury, B., \& Engle Merry, S. (2012). Indicators as a Technology of Global Governance. Wiley on behalf of the Law and Society Association, 46(1), 71-104.

Del Popolo, F., Oyarce, A. M., \& Ribotta, B. (2009). Indígenas urbanos en América Latina: Algunos resultados censales y su relación con los Objetivos de Desarrollo del Milenio. Notas de población, 86.

Díaz Montenegro, E., Rojas Curieux, T., \& Gonzáles Castaño, G. (2011). Namoi nam trik pesenamitan: Reflexiones sobre el proceso de revitalización de la lengua nam trik de Totoró, Cauca, Colombia. STLILLA-2011, 1-14.

Duchêne, A., \& Heller, M. (Ed.). 2008. Discourses of endangerment: Ideology and interest in the defence of languages. London; New York: Continuum. 
Fishman, J. A. (1991). Reversing Language Shift: Theoretical and Empirical Foundations of Assistance to Threatened Languages. Clevedon: Multilingual Matters. García, O., Flores, N., \& Spotti, M. (2016). Introduction Language and Society. A Critical Poststructuralist Perspective. En García, O., Flores, N. y Spotti, M. (Ed.), Bilingualism, Multilingualism, Globalization, and Superdiversity (pp. 1-16). Oxford University Press.

Gumperz, J. J. (1964). Linguistic and social interaction in two communities. American Anthropologist, 66(6), 137-153.

Hornberger, N. H., \& Coronel-Molina, S. M. (2004). Quechua language shi$\mathrm{ft}$, maintenance, and revitalization in the Andes: The case for language planning. International Journal of the Sociology of Language, 167, 9-68.

Jørgensen, J. N., Karrebaek, M. S., Madsen, L. M., \& Møller, J. S. (2011). Polylanguaging in Superdiversity. Diversities, 2(13), 22-37.

Kroskrity, P. V. (2006). Language Ideologies. En Duranti, A. (Ed.), A Companion to Linguistic Anthropology (pp. 496-517). Malden: Blackwell Publishing.

Lara, R. A. (2013). Informe parcial Cabildos indígenas urbanos. Ministerio del Interior. Dirección de Etnias- Subdirección de Asuntos Indígenas. Bogotá.

Léglise, I. (2013). Multilinguisme, variation, contact. Des pratiques langagières sur le terrain à l'analyse de corpus hétérogènes (HDR Dissertation, Institut National des Langues et Civilisations Orientales). Recuperado de: http:// hal.archives-ouvertes.fr/tel-00880500/

(2017). Multilinguisme et hétérogénéité des pratiques langagières. Nouveaux chantiers et enjeux du Global South. Langage et société, 160-161, 251-266. (2018). Pratiques langagières plurilingues et frontières de langues. En Auzanneau, M. y Greco, L. (Ed.), Dessiner les frontières (pp. 143-169). Paris: ENS Editions.

López, L. E., \& Wolfgang, K. (1999). La educación intercultural bilingüe en América Latina: Balance y perspectivas. Revista Iberoamericana de Educación, 20, 17-84.

Makoni, B. (2017). Urban Languages in African Contexts. Toward a Multimodal Approach to Urban Languages. En García, O., Flores, N. y Spotti, M. (Eds.), The Oxford Handbook of Language and Society (pp. 281-298). https://doi.org/10.1093/oxfordhb/9780190212896.001.0001

Makoni, S., \& Pennycook, A. (2005). Disinventing and (Re)Constituting Languages. Critical Inquiry in Language Studies, 2(3), 137-156. https://doi. org/10.1207/s15427595cils0203_1

Martín Rojo, L. (2015). The Social Construction of Inequality in and through Interaction in Multilingual Classrooms. En Markee, N. (Ed.), The Han- 
dbook of Classroom Discourse and Interaction (pp. 490-505). https://doi. org/10.1002/9781118531242.ch29

Martínez Bocanegra, D. (2008). El pueblo rom-gitano que habita la ciudad de Bogotá. Bogotá: Misión Rural.

Meissner, F., \& Vertovec, S. (2015). Comparing super-diversity. Ethnic and Racial Studies, 38(4), 541-555. https://doi.org/10.1080/01419870.2015.980295

Molina Echeverri, H. (2007). Nuevos escenarios de vida indígena urbana: El caso de Bogotá. Etnias \& política, 4, 16.

Morrell, E., \& Duncan-Andrade, J. (2004). What they do learn in school: HipHop as a bridge to canonical poetry. En Mahiri, J. (Ed.), What They Don't Learn in School: Literacy in the Lives of Urban Youth (pp. 247-268). New York: Peter Lang.

Motta González, N. (2004). Con chirimías, lanas y medicinas: Hombres y mujeres indígenas reinventando el cabildo en la ciudad. En Castellanos, G. (Ed.), Textos y Prácticas de Género. Cali: Centro de Estudios de Género Mujer y Sociedad. Universidad del Valle.

Motta González, N., \& Posso, J. (2007). Hacia el reconocimiento de una identidad indígena urbana en Cali. Estudio etnológico de las comunidades indigenas ubicadas en el municipio de Santiago de Cali, Kofán, Guambiana, Quichua, Inga, Yanacona, Nasa. Cali: Alcaldía Santiago de Cali; Ministerio del Interior y de Justicia y Fundación General de Apoyo.

Nussbaum, L. (2012). De las lenguas en contacto al habla plurilingüe. En Unamuno, V. y Maldonado, A. (Ed.), Prácticas y repertorios plurilingües en Argentina (pp. 273-284). Bellaterra: Grup de Recerca en Ensenyament i Interacció Plurilingües Universitat Autònoma de Barcelona.

Ordoñez, J. T., Colmenares, F. A., Bernal, R., \& Gincel, A. (2014). Migraciones de los Kichwas-Otavalo en Bogotá. Revista de Estudios Sociales, 48, 43-56. http://dx.doi.org/10.7440/res48.2014.04

Ospina Bozzi, A. M. (2015). Mantenimiento y revitalización de lenguas nativas en Colombia. Reflexiones para el camino. Forma y Función, 28(2), 11-48. https://doi.org/10.15446/fyf.v28n2.53538

Otheguy, R., García, O., \& Reid, W. (2015). Clarifying translanguaging and deconstructing named languages: A perspective from linguistics. Applied Linguistics Review, 6(3), 281-307. https://doi.org/10.1515/ applirev-2015-0014

Pancho, A., \& Navas, C. (2007). El derecho a la educación en el municipio resguardo de Jambaló (departamento del Cauca). La paradoja de la educación indígena. En Rey, E. (Ed.), Indígenas sin derechos. Situación de los 
derechos humanos de los pueblos indígenas (pp. 287-306). Bogotá: Centro de Cooperación al Indígena: Organización Indígena de Antioquia.

Rampton, B. (2006). Language in late modernity: Interaction in an urban school. Cambridge: Cambridge University Press.

Rey, E. (2007). ¿Qué hay detrás de un número? Análisis de los “síntomas" sobre la situación de los pueblos indígenas desde una lectura del Censo de 2005. En Rey, E. (Ed.), Indígenas sin derechos. Situación de los derechos humanos de los pueblos indígenas (pp. 151-169). Bogotá: Centro de Cooperación al Indígena: Organización Indígena de Antioquia.

Rincón Fagua, D., \& Torres Padila, D. (2019). Propuesta para un diagnóstico sociolingüístico de poblaciones minorizadas en Cartagena. Aproximación a su diversidad lingüística y sociocultural. Visitas al Patio, $O(13), 61-80$. https://doi.org/10.32997/2027-0585-vol.0-num.13-2019-2315

Rojas, A., \& Castillo, E. (2005). Educar a los Otros. Estado Políticas educativas y diferencia cultural en Colombia. Popayán: Editorial Universidad del Cauca.

Romaine, S. (2000). Language in Society: An Introduction to Sociolinguistics (Second Edition). Oxford, New York: Oxford University Press.

Sánchez Moreano, S. (2018). Negociación de posicionamientos sociales (stances) a través del debilitamiento del fonema /s/ en el español hablado por los quichuas ecuatorianos en Cali (Colombia). Pragmática Sociocultural / Sociocultural Pragmatics, 6(1), 33-70. https://doi.org/10.1515/ soprag-2017-0019

(2019). Repertorios lingüísticos y representación de la diversidad lingüística en Leticia (Colombia) y su periferia. Visitas al Patio, 13, 12-36. https://doi.org/10.32997/2027-0585-vol.0-num.13-2019-2313

Sierra Martínez, L. M., Rojas Curieux, T., Meza Vega, E., Cobos Lozada, C. A., Villegas González, J. A., \& Solarte Camayo, E. A. (2015). Construcción de materiales educativos que apoyan la enseñanza del nasa yuwe. Popayán: Editorial Universidad del Cauca.

Sinigüi Ramírez, S. Y. (2007). Es posible ser indígena en la ciudad. Sobre estudios indígenas y afrocolombianos. Memoria personal. Revista de Educación y Pedagogía, 49, 199-214.

Spotti, M., \& Blommaert, J. (2017). Bilingualism, Multilingualism, Globalization, and Superdiversity: Toward Sociolinguistic Repertoires. En García, O., Flores, N. y Spotti, M. (Ed.), The Oxford Handbook of Language and Society (pp. 161-178). Oxford: Oxford University Press.

Thomason, S., \& Kaufman, T. (1988). Language Contact, Creolization, and Genetic Linguistics. Berkeley, Los Angeles: University of California Press. 
Torres, A. (Ed.). (2010). Niñez indigena en migración: Derechos en riesgo y tramas culturales. Quito: FLACSO, UNICEF, AECID.

United Nations Housing Rights Programme (2010). Urban Indigenous Peoples and Migration: A Review of Policies, Programmes and Practices (No 8; p. 214). Nairobi: United Nations.

Usma Wilches, J. A., Ortiz Medina, J. M., \& Gutiérrez, C. (2018). Indigenous Students Learning English in Higher Education: Challenges and Hopes. Íkala, Revista de Lenguaje y Cultura, 23(2), 229-254.

Valencia Galvis, R. (2012). Comunidad quechua en Medellín: Lengua, identidad y cultura. Boletín de Antropología Universidad de Antioquia, 27(44), 304-318.

Vertovec, S. (2007). Super-diversity and its implications. Ethnic and Racial Studies, 30(6), 1024-1054.

(2009). Transnationalism. London, New York: Routledge.

Winford, D. (2003). An Introduction to Contact Linguistics. Oxford: Blackwell. 